\title{
Strain-energy transport during fracture of metallic glasses
}

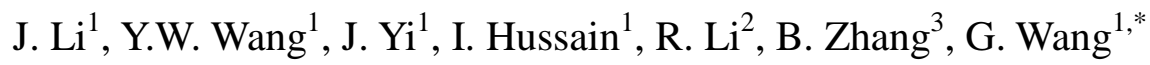 \\ ${ }^{1}$ Laboratory for Microstructures, Institute of Materials, Shanghai University, Shanghai 200444, \\ China \\ ${ }^{2}$ School of Materials Science and Engineering, Beihang University, Beijing 100709, China \\ ${ }^{3}$ School of Materials Science and Engineering, Hefei University of Technology, Hefei 230009, \\ China \\ *Corresponding author: g.wang@ shu.edu.cn
}

\begin{abstract}
In a strained metallic glass (MG), depending on its fracture toughness, the failure mode can be dominated by shear-banding failure (for tough MGs) or cracking failure (for brittle MGs). During the fracture process, crack formation and propagation create a nonlinear strain field at crack tip. The transportation of the strain energy stored in the strained MG to the crack tip is of importance in understanding the deformation mechanism of MGs. By using digital image correlation method, we perform direct and precise measurements of the strain concentration, including maximum strain value and the strain-energy distribution, in four MGs. After yielding, plastic deformation is localized into shear bands or crack tip in a scale of micrometer, which means a systematic transition from the linear elastic behavior to the strongly nonlinear behavior. Through quantitatively evaluating the balance between the fracture energy and the strain-energy density, the failure modes are discussed based on a hyperelasticity model. This result provides a comprehensive picture of how remotely applied forces drive MGs failure in the fundamentals of fracture states.
\end{abstract}

Key words: In-situ observation; DIC technique; metallic glasses; hyperelasticity model; crack formation 


\section{Background}

Metallic glasses (MGs) without crystallographic defects acting as the plastic medium, usually have a high strength and a limited plasticity [1-3]. After the elastic deformation, some MGs with high toughness, such as $\mathrm{Zr}$ - and $\mathrm{Cu}$-based MGs [4,5], yield through shear-band sliding, and some MGs with low toughness, such as Mg-based and La-based MGs [6,7], exhibit a macroscopic fracture without the activation of plastic yielding. Therefore, the relationship between the toughness and the plastic yielding via shear banding in MGs is of importance to understand elasto-plastic deformation mechanism. So far, most of work focus on the plastic mechanism, which generated numerous theories to quantitatively describe the deformation mechanism of MGs, such as the shear transformation zone (STZ) theory [8,9], the free volume theory [10], the flow units model [11, 12], the liquid-like zone theory [13], and the geometrically unfavored motifs (GUMs) model [14] etc. Furthermore, the correlation between the fracture toughness and the elastic parameters of MGs was intensively studied to elucidate the deformation process [15-21]. However, the fracture of MGs is actually a process of a crack formation and propagation at a small scale [22]. The existence of a crack can dramatically amplify the external stress [23], in which a strain-concentration field is formed through a transportation of the externally applied elastic energy to the crack tip [24]. How the elastic energy transports in the shear-banding process of MGs, and how the elastic energy dissipates in the fracture process without plastically shear banding have not been well theoretically depicted because directly evaluating the elastic-energy accumulation is difficult. The key to understanding these questions may lie in the structure of the region in the front of the crack tip [25, 26], where strains become so large that the linear elastic stress-strain response of MGs is already broken down.

Digital image correlation (DIC) technique provides an effective method to map the local displacement and the strain distribution on the sample's surface through the computation of a series of images recorded during the in-situ deformation [27, 28]. Both a full-field visualization, and a dynamic evaluation of the strain are profiled simultaneously on a local level. The DIC technique has been previously applied to investigate the strain localization behavior, such as shear banding in MGs [29-31], whisker growth [32], crack growth in ceramics [33], damage measurement in rocks [34] etc. In these work, the DIC technique could accurately estimate the local strength of materials [35], locate the initiation place of micro-crack [36], and evaluate the maximum strain value for failure [37]. 
Accordingly, in this paper, four MGs, i.e., $\mathrm{Zr}_{52.5} \mathrm{Cu}_{17.9} \mathrm{Ni}_{14.6} \mathrm{Al}_{10} \mathrm{Ti}_{5}$ (at.\%), $\mathrm{Ce}_{70} \mathrm{Ga}_{8} \mathrm{Cu}_{22}$, $\mathrm{Mg}_{65} \mathrm{Cu}_{25} \mathrm{Gd}_{10}$ and $\mathrm{Co}_{59} \mathrm{Ta}_{8} \mathrm{~B}_{33}$ with different mechanical properties are chosen as the model materials. With the deformation evolving from an elastically stressing to a plastically yielding in MGs, a correlation between the strain-energy density and the fracture energy is observed to explain the failure modes.

\section{Experimental procedure}

The $\mathrm{Zr}_{52.5} \mathrm{Cu}_{17.9} \mathrm{Ni}_{14.6} \mathrm{Al}_{10} \mathrm{Ti}_{5}$ and $\mathrm{Ce}_{70} \mathrm{Ga}_{8} \mathrm{Cu}_{22}$ alloys were prepared by arc melting appropriate amounts of pure metal elements, with purities higher than $99.99 \%$, in an argon atmosphere, and followed by suction casting into $\mathrm{Cu}$-mould to form plate and rod samples. The $\mathrm{Mg}_{65} \mathrm{Cu}_{25} \mathrm{Gd}_{10}$ metallic glass was prepared by induction melting plus injection casting. $\mathrm{Cu}-\mathrm{Gd}$ pre-alloy was prepared by arc melting the mixture of pure metal elements in the argon atmosphere. A mixture of pure $\mathrm{Mg}$ element and $\mathrm{Cu}-\mathrm{Gd}$ pre-alloy was induction melted under the argon atmosphere and then injected into a copper mould to form rod samples. The $\mathrm{Co}_{56} \mathrm{Ta}_{9} \mathrm{~B}_{35}$ glass rods were also prepared by induction melting casting. Co-B pre-alloy was prepared by arc melting the mixture of pure elements in the argon atmosphere. A mixture of pure Co, Ta element and Co-B pre-alloy were prepared by copper mould casting in the argon atmosphere to form rod samples. The diameter of the $\mathrm{Zr}_{52.5} \mathrm{Cu}_{17.9} \mathrm{Ni}_{14.6} \mathrm{Al}_{10} \mathrm{Ti}_{5}$ (Zr-based MG), $\mathrm{Ce}_{70} \mathrm{Ga}_{8} \mathrm{Cu}_{22}$ (Ce-based MG), $\mathrm{Mg}_{65} \mathrm{Cu}_{25} \mathrm{Gd}_{10}(\mathrm{Mg}$-based MG) and $\mathrm{Co}_{59} \mathrm{Ta}_{8} \mathrm{~B}_{33}$ (Co-based MG) glassy rods were $2 \mathrm{~mm}$.

The glassy phase structures of each cast specimen were examined by X-ray diffraction (XRD) in a Rigaku DLMAX-2550 diffractometer with the $\mathrm{Cu}-\mathrm{K}_{\alpha}$ radiation $(\lambda=0.1542 \mathrm{~nm})$. The fractography of the fractured metallic glasses were observed with an APHENOM ${ }^{\mathrm{TM}}$ G2 (FEI company) scanning electron microscope (SEM). Compression specimens with a length/width (or length/diameter) ratio of 2 were cut from the rod samples. The ends of each specimen were carefully ground to a surface roughness of less than $1 \mu \mathrm{m}$. The rod samples were ground along the loading direction to be a half cylinder, as shown in the inset of Fig. 2a. After grinding, a surface of the cross-section with a size of $4 \mathrm{~mm} \times 2 \mathrm{~mm}$ was shaped. In the center of the surface of the cross-section, a small area with a size of $2 \mathrm{~mm} \times 1 \mathrm{~mm}$ (rectangular region covered by red color in the inset of Fig. 2a) was used for white digital speckle image correlation (DIC) method observation. Compression tests were conducted using an MTS CMT5205 machine at room temperature and a strain rate of $2.5 \times 10^{-4} \mathrm{~s}^{-1}$. For each MG, the compression tests were repeated four times to exclude 
the occasional results. The elastic parameters including elastic modulus, shear modulus, bulk modulus and shear wave speed were measured by the ultrasonic technique, for which an Olympus Panametrics NDT 5900PR ultrasonic testing device was used. The results are listed in Table I. The stress intensity factor, $K$, measurements were carried out through using the circumferentially notched bar [17], which was estimated to be, $K=\left(0.526 P D^{0.5}\right) / d^{2}$, where $D$ is the diameter of the rods used for the stress intensity factor $(2 \mathrm{~mm}), P$ is the applied load, $d$ is the diameter of circumferential notch, and $1.2 \leq D / d \leq 2.1$. The measured stress intensity factors of four MGs are listed in Table I.

The strain field of the MGs' surfaces was observed by the DIC method. The surface of the sample was firstly spray-painted to generate artificial speckles and then illuminated by two fiber optic white light sources during loading. A CCD video camera (JAI CV-A1) was placed in front of the spray-painted surface to acquire the sequential speckle images necessary for performing digital speckle correlation. During the loading process, consecutive surface images were recorded with a $831 \times 564$ pixel CCD array and a length-pixel ratio of approximately $4.75 \mu \mathrm{m} /$ pixel. The sequential images acquiring frequency was $4 \mathrm{~Hz}$. A $211 \times 422$ pixel $(1 \times 2 \mathrm{~mm})$ calculated domain was located at the middle of the sample. An image was obtained prior to loading that served as the reference (un-deformed) condition. The sequential images were analysed with respect to this reference condition using DIC, that can provide a displacement resolution in 0.01 subpixels, i.e., approximately $0.1 \mu \mathrm{m}$ by a combination of the sub-pixel technique and the Newton-Raphson iteration algorithm. The strain distribution at different loads could then be identified by a series of pronounced contour maps. The DIC observation for each MG was repeated three times to confirm that the results were reproducible.

\section{Results}

Usually, the deformation behavior of MGs is seriously influenced by the microstructure of glassy phase. Especially, for the MGs with a relative low glass-forming ability, the microstructure of the glassy phase could be significantly changed during the quenching process. In the present study, the structures of four MGs were examined by XRD, which do not show any sharp diffraction peaks, confirming the glassy nature of four MGs (see Fig. 1). Therefore, the influence of the structure of the MGs on the mechanical tests can be ignored.

The nominal compression stress-strain curves of four MGs are plotted in Fig. 2. Their yield 
strength and plastic strain are listed in Table I. The Zr-based MG shows the largest compressive ductility in the four MGs, which is manifested as a series of serrated events (Fig. 2a). The Ce-based MG shows a slightly plastic yielding after the elastic stage, but without obviously plastic flow plateau as shown in the Zr-based MG (Fig. 2b). The Mg-based MG shows a linearly elastic deformation, and then followed by a catastrophic fracture (Fig. 2c). The deformation of the Co-based MG is also composed by the elastic deformation, and the plastic yielding plateau (Fig. 2d). All of the mechanical properties of the MGs are listed in Table I. It needs to point out that the plastic deformation of the Ce-based MG exhibits a strong strain-hardening behavior. The glass-transition temperature of the Ce-based $\mathrm{MG}$ is $363 \mathrm{~K}$ [38] that is very close to room temperature $(303 \mathrm{~K})$. In this case, the deformation of the Ce-based MG possibly shows a homogeneous plastic flow, depending on the strain rate [39]. In the present study, the plastic strain of the Ce-based MG is contributed from the homogeneous deformation, which causes a significant strain-hardening behavior. When the deformation approaches the maximum stress, several serrated flow occurs, which indicates that several shear bands are formed before the fracture.

The fractured MGs are observed by SEM. Based on the fractographies shown in Fig. 3, the failure mechanisms can be classified into two categories. For the $\mathrm{Zr}$ - and Ce-based MGs, we can see a significant shear-fracture behavior. The fracture angles, i.e., the angle between the fracture surface and the loading direction, of the $\mathrm{Zr}$ - and Ce-based MGs are $42^{\circ}$ and $52^{\circ}$, respectively, which suggests that the shear-banding mechanism dominates the plastic deformation (Figs. 3a and c). Some shear bands appear on the lateral surface of the fractured $\mathrm{Zr}$ - and Ce-based MGs (the insets of Figs. $3 \mathrm{~b}$ and $3 \mathrm{~d}$ ) which correspond to the serrated flow behavior in the stress-strain curves of the $\mathrm{Zr}$ and Ce-based MGs. After the plastic deformation, the crack can form along a major shear band (shear plane) to cause the final shear separation. The fracture surfaces of the $\mathrm{Zr}$ - and Ce-based MGs exhibit the vein-like pattern, indicating that the viscous-flow layer is formed during the shear fracture (Figs. 3b and d). In this case, the failure mode of the $\mathrm{Zr}$ - and Ce-based MGs is shear-banding failure. The Co- and Mg-based MGs are split into several pieces, and the fracture behavior does not show the shear separation upon the compressive stress (Figs. 3e and g). The fracture angle of the Mg-based MG could be roughly $90^{\circ}$ (Fig. 3e). The fracture surface of the $\mathrm{Mg}$ and Co-based MGs show mirror-like morphology characterized as a periodic corrugation pattern in the nanoscale (Figs. $3 \mathrm{f}$ and h). It is evident that the fracture of the Mg-and Co-based MGs without the significant formation of shear band. Therefore, the Mg- and Co-based MGs exhibit a cracking 
failure mode.

The two different failure modes might be attributed to the strain evolution in the samples. To address this, a strain-field evolution is investigated by DIC. The speckle images of the four MGs (Figs. 4a, 5a, 6a and 7a) show homogeneously scattering white particles without the stress. According to the speckle image, the strain-field evolutions can be calculated, including the linear-strain $\left(\varepsilon_{x}\right)$ fields along the expansion direction, i.e., $x$-axis, the linear-strain $\left(\varepsilon_{y}\right)$ fields along the loading direction, i.e., $y$-axis, and the shear-strain $\left(\gamma_{x y}\right)$ fields. The maximum linear strain in $x$ and $y$-axis, i.e., $\varepsilon_{x \max }$, and $\varepsilon_{y \max }$, and the maximum shear strain, $\gamma_{\max }$, are deduced by the following equation [40],

$$
\left\{\begin{array}{c}
\varepsilon_{x \max }=\frac{1}{2}\left[\varepsilon_{x}+\varepsilon_{y}-\sqrt{\left(\varepsilon_{x}-\varepsilon_{y}\right)^{2}+\gamma_{x y}{ }^{2}}\right] \\
\varepsilon_{y \max }=\frac{1}{2}\left[\varepsilon_{x}+\varepsilon_{y}+\sqrt{\left(\varepsilon_{x}-\varepsilon_{y}\right)^{2}+\gamma_{x y}{ }^{2}}\right] \\
\gamma_{\max }=\sqrt{\left(\varepsilon_{x}-\varepsilon_{y}\right)^{2}+\gamma_{x y^{2}}}
\end{array}\right.
$$

The contour maps of the maximum strain fields, including the $\varepsilon_{x \max }$ field, the $\varepsilon_{y \max }$ field, and the $\gamma_{\max }$ field, of the Zr-based MG at different stresses are plotted in Figs. 4b, c and d, respectively. It can be seen that in the stress range from $19 \mathrm{MPa}$ to $1410 \mathrm{MPa}$, the strain concentrations are marginal in three fields. When the stress is further increased to $1749 \mathrm{MPa}$, i.e., the yield strength, the strain concentration appears in the stress range from 1410 to $1749 \mathrm{MPa}$. Especially, in the $\varepsilon_{y \max }$ and $\gamma_{\max }$ fields, some high strain-concentration striations are formed. After yielding, with increasing the plastic strain, the maximum strains are further increased while the stress does not significantly increase, which is attributed to the formation and propagation of shear bands. These strain-concentration striations almost distribute at a shear angle of $45^{\circ}$, as marked in Fig. 4.

Figure 5 shows the DIC images measured from the Ce-based MG. In the stress range from 34 $\mathrm{MPa}$ to $274 \mathrm{MPa}$, the contour maps do not show significant strain concentration in three fields (Figs. $4 \mathrm{~b}, \mathrm{c}$ and d). When the stress approaches the yield stress of approximately $403 \mathrm{MPa}$ and is higher than the yield stress $\left(569 \mathrm{MPa}\right.$ and $586 \mathrm{MPa}$ ), the strain concentration is raised. In the $\varepsilon_{x \max }$, and $\varepsilon_{y \max }$ fields, the strain-concentration striations are formed. In the $\gamma_{\max }$ field, the strain-concentration striation is not significant. After yielding, with increasing the plastic strain, the strain concentration is more significant than the case in the elastic stage, which suggests that the inhomogeneous deformation also occurs although the homogeneous deformation already influences the plastic strain. 
The strain-concentration striations are orientated to a shear plane with the fracture angle of approximately $63^{\circ}$ (Figs. $5 \mathrm{c}$ and d), which is close to the fracture angle of $52^{\circ}$ observed in Fig. 3c. The possible reason is that the homogeneous deformation causes the sample to expand along the direction being perpendicular to the loading direction, which may distort the strain-concentration striation to deviate the shear plane at the shear angle of $45^{\circ}$.

Figure 6 shows the DIC images measured from the Co-based MG, which also includes the $\varepsilon_{x \max }, \varepsilon_{y \max }$ and $\gamma_{\max }$ fields in Figs. $6 \mathrm{~b}, \mathrm{c}$ and d, respectively. It is evident that when the stress is less than $2052 \mathrm{MPa}$, the strain concentration can be ignored. When the stress is higher than $2052 \mathrm{MPa}$, which is well below the yield stress $4763 \mathrm{MPa}$, the strain concentration starts, especially in the $\varepsilon_{y \max }$ and $\gamma_{\max }$ fields (Figs. $6 \mathrm{c}$ and d). With the stress increasing to the yield stress of $4829 \mathrm{MPa}$, besides the concentrated strain value increases, the strain-concentration striations are formed in the $\varepsilon_{x m a x}$, $\varepsilon_{y \max }$ and $\gamma_{\max }$ fields (Figs. 6b, c and d).

The DIC images of the Mg-based MG are mapped in Fig. 7. When the stress is less than 458 MPa that is well below the yield strength of $895 \mathrm{MPa}$, the strain concentration is insignificant. However, from approximately $458 \mathrm{MPa}$, the strain concentration comes to work with increasing stress. The strain-concentration striations are observed in the $\varepsilon_{y \max }$ and $\gamma_{\max }$ fields when the stress is higher than $709 \mathrm{MPa}$.

To further characterize the strain concentration evolving with the stress, the profile of the distributions of the maximum $\varepsilon_{x \max }, \varepsilon_{y \max }$ and $\gamma_{\max }$ values at different stresses of four MGs on the maximum shear stress plane, i.e., along the dash lines in Figs. 4-7 are plotted in Fig. 8. It can be seen that the strain values of $\varepsilon_{x \max }, \varepsilon_{y \max }$ and $\gamma_{\max }$ increase with increasing the stress.

\section{Discussion}

After yielding, the Zr- and Ce-based MGs shows the occurrence of plastic flow through shear bands formation and propagation, and the Mg- and Co-based MGs fracture catastrophically. The influence of the stiffness of the compression-testing machine on the plastic deformation of the MGs is required to be considered. It is well known that the release of the elastic-energy stored in the testing machine can seriously influence the instability of shear band [41]. After yielding, the elastic energies, including the energy stored in the MGs and the energy stored in the testing machine, are released to form the shear bands. The stiffness of the testing machine determines the quantity of the 
elastic energy stored in the testing machine. A shear-band instability index (SBI) of $U\left[U=\frac{\pi E d}{4 \rho \kappa_{M}}\right.$, (where $\kappa_{M}$ is the stiffness of the testing machine, $E, d$ and $\rho$ are the elastic modulus, diameter and aspect ratio (height to diameter ratio) of the sample, respectively] is introduced to quantitatively describe the influence of the stiffness of the testing machine on shear banding. In the present study, the $\kappa_{M}$ value is a constant because all of the compression tests were carried out in one machine. The $d$ and $\rho$ values are also the constant due to the same geometric size of the four MGs. Thus, the $E$ value dominates the $U$ value. We assume the $U$ value of the Ce-based MG to be a unit. Based on the elastic moduli listed in Table I, the $U$ values of the Ce-based MG, Mg-based MG, the Zr-based MG, and the Co-based MG are 1, 1.66, 2.90, and 7.87, respectively. It is obvious that the $U$ value of the Co-based MG is the largest one, which suggests that the shear-band instability is most unstable in the four MGs. Oppositely, shear banding in the Ce-based MG is the most stable case, which means that the plastic deformation via plastic shearing in the Ce-based MG is the largest in the four MGs. The plasticity of the Mg-based MG also should be very good due to its relative lower $U$ value of 1.66. However, the stress-strain behaviors in Fig. 2 suggest that the plasticity of the Zr-based MG is the largest one. Therefore, the stiffness of the testing machine is not the main reason dominating shear banding and the shear fracture. Therefore, it requires to further characterize the crack-formation process.

The DIC images provide the information of the strain distribution, and also the strain energy distribution. The width of the strain-concentration striation, $l_{y}$, marked in Figs. 4-7, shows that the average widths of the striations in the $\mathrm{Zr}$-, Ce-, Co- and Mg-based MGs are $364 \pm 84 \mu \mathrm{m}, 586 \pm 99$ $\mu \mathrm{m}, 385 \pm 79 \mu \mathrm{m}$, and $555 \pm 101 \mu \mathrm{m}$, respectively. The formation of these strain-concentration striations suggests that the elastic energy stored in the MGs distributes inhomogeneously at least at the yield point. In the yielding plasticity, these strain-concentration striations act as the original place of shear banding for the ductile MGs, or cracking for the brittle MGs, which relax the strain accommodation in these striations. At the fracture moment, the crack must be formed in the strain-concentration striation or in the shear bands. Therefore, the crack formation, i.e., the fracture process, should be associated with the dissipation of the strain energy in the strain-concentration striations.

The Irwin-Orowan small-scale zone model [42] has clearly shown that the fracture surface separation processes are confined to a small zone in the front of the crack tip, defined as the fracture 
process zone with a radius of $R$ (Fig. 9). The radius of the fracture process zone, $R$, for plane strain can be estimated as,

$$
R=\frac{1}{6 \pi}\left(\frac{K}{\sigma_{y}}\right)^{2} .
$$

where $K$ is the stress intensity factor $\left(\mathrm{MPa} \cdot \mathrm{m}^{0.5}\right)$, and $\sigma_{y}$ is the yield strength. Accordingly, the $R$ values for the Zr-, Ce-, Co-, and Mg-based MGs are $31 \mu \mathrm{m}, 10 \mu \mathrm{m}, 59 \mathrm{~nm}$ and $112 \mathrm{~nm}$, respectively. The $R$ values are almost the same of the characteristic size, $W$, (i.e., the vein pattern on the fracture surface of the $\mathrm{Zr}$ - and Ce-based MGs, and the spacing values of the periodic corrugations on the fracture surface of the Co- and Mg-based MGs, Fig. 3). Therefore, the fractographies give the solid evidences to confirm the formation of the fracture process zone in the four MGs.

Once the crack commences to penetrate into the glassy phase, a viscous fluid meniscus will be formed in the fracture process zone due to adiabatic heating and the shear dilatation [43-45]. In this case, a nonlinear behavior will occur when the material is failing at the tip of the crack due to the extreme deformation condition, such as high crack propagation speed, and high adiabatic heating, etc. [46]. Furthermore, regarding the Mg- and Co-based MGs that are broken into several pieces, the dynamical instability of crack propagation should be considered because of the crack bifurcation due to the high crack propagation speed [47]. Therefore, a hyperelasticity model was proposed to depict this nonlinear behavior in the dynamic crack tip [48], in which the instability of the dynamic crack propagation is dominated by a competition between a stiffening behavior and a softening behavior [49]. A radius of the hyperelastic region, $r_{H}$, and a characteristic length for local energy flux, $\gamma$, is used to characterize the fracture energy balance process, which is shown in Fig. 9 [48]. The characteristic length for the local energy flux represents an elastic-energy storing region around the crack tip. From this region, strain energy flows to the moving crack. Therefore, a ratio of $r_{H} / \gamma$ is used to characterize the competition between the stiffening behavior and softening behavior. Once the $r_{H}$ value is comparable to the $\gamma$ value, hyperelastic softening dominates crack instability, which means that the required energy to sustain crack moving can be transported from the hyperelastic region. On the other hand, if the $r_{H}$ value is far less than the $\gamma$ value, i.e., $r_{H} / \gamma \rightarrow 0$, the crack instability is governed by elastically stiffening. In this case, the hyperelastic region cannot provide enough energy to push the crack tip penetrating into the glassy phase because the size of the hyperelastic region is too small to be ignored. More energy flows into the crack tip from the linear elastic region to sustain crack propagation as compared to that in elastic softening [46]. As such, the 
characteristic length of local energy flux becomes more significant.

In the present study, regarding that the viscous fluid is formed in the fracture process zone, we can rationally treat the fracture process zone as a nonlinear hyperelastic region, suggesting that the $r_{H}$ value is equivalent to the $R$ value. Since the elastic energy stored in the strain-concentration striation will be transported to the crack formation during the fracture stage, the characteristic lengths for local energy flux is believed to be associated with the average width of strain-concentration striation, $l_{y}$. Therefore, the $2 \gamma\left(=l_{y}\right)$ value of the $\mathrm{Zr}-, \mathrm{Ce}-, \mathrm{Co}-$ and $\mathrm{Mg}$-based MGs are $364 \pm 84 \mu \mathrm{m}, 586 \pm 99 \mu \mathrm{m}, 385 \pm 79 \mu \mathrm{m}$, and $555 \pm 101 \mu \mathrm{m}$, respectively. The characteristic length for the local energy flux reflects a region stored linear elastic energy around the crack tip. From this region, the strain energy flows to the moving crack, which is schematically shown in Fig. 9. The ratios of $r_{H} / \gamma$ for the $\mathrm{Zr}-, \mathrm{Ce}-, \mathrm{Co}-$, and $\mathrm{Mg}$-based MGs are then calculated to be $1.7 \times 10^{-1}, 3.4 \times 10^{-2}, 1.5 \times 10^{-4}$ and $4.9 \times 10^{-4}$, respectively. It is evident that the $r_{H} / \gamma_{Z r}$ value of $1.7 \times 10^{-1}$, and the $r_{H} / \gamma_{C e}$ value of $3.4 \times 10^{-2}$ suggest that the characteristic lengths of local energy flux for the $\mathrm{Zr}$ - and Ce-based MGs can be comparable to the size of the hyperelastic region, while the $r_{H} / \gamma_{C o}$ value of $1.5 \times 10^{-4}$ and the $r_{H} / \gamma_{M g}$ value of $4.9 \times 10^{-4}$ suggest that the local energy flux of the Co- and Mg-based MGs becomes the dominative source providing the energy to support the crack propagation. Thus, the failure mode of the $\mathrm{Zr}$ - and Ce-based MGs is manipulated by the hyperelastic softening mechanism, and that of the Co- and Mg-based MGs is dominated by the linear elastic stiffening mechanism.

Whether the hyperelastic softening mechanism or the linear elastic stiffening mechanism must be associated with an energy balance process that dominates the crack propagation. According to the Irwin relationship, fracture energy, $\Gamma$, in crack propagation is expressed as [50]:

$$
\Gamma=\frac{1-v^{2}}{E}\left(1-\frac{V}{V_{R}}\right) K^{2},
$$

where $v$ is the Poisson ratio, $V$ is the crack propagation speed, and $V_{R}$ is the Rayleigh wave speed. Usually, in MGs, $V_{R} \approx 0.9225 c_{s}$, where $c_{s}$ is the shear wave speed [51], and the maximum crack propagation cannot be larger than $0.73 V_{R}$. The shear wave speeds of four MGs are listed in Table I. Accordingly, the fracture energies of four MGs as functions of the crack propagation speed are plotted in Fig. 10. Since the fracture will occur preferentially in the strain-concentration region, the strain energy in the strain-concentration striation will be transported to be the fracture energy. The strain energy density, $S$, in the strain-concentration striation of the $\varepsilon_{y}$ field can be estimated by [50], 


$$
S=\frac{1}{2} E \varepsilon_{y}^{2} l_{y} /\left(1-v^{2}\right)
$$

where $l_{y}$ is the width of the strain-energy storage range. In the present study, the $l_{y}$ value is the width of the strain-concentration striation in the $\varepsilon_{y}$ field. After integrating the strain value covered by the strain-concentration striation peak that is marked in Fig. 8, we can evaluate the strain-energy densities of the four MGs, which are shown in Fig. 10. For the Zr-based MG, the strain-energy density, $S_{Z r}$, is close to the low limit of the fracture energy, $\Gamma_{Z r}$. The $S_{C e}$ value is 1.7 times higher than the fracture energy of the Ce-based $\mathrm{MG}, \Gamma_{C e}$. For the Co- and $\mathrm{Mg}$-based MGs, the strain-energy densities are 100 times and 5 times larger than the $\Gamma_{C o}$ and $\Gamma_{M g}$ values, respectively. It is clear that the strain-energy densities of the $\mathrm{Zr}$ - and Ce-based MGs are comparable to the fracture energies, while the strain-energy densities of the $\mathrm{Co}$ - and $\mathrm{Mg}$-based $\mathrm{MGs}$ are much larger than the fracture energies.

Since that the hyperelastic softening mechanism dominates the crack propagation in the $\mathrm{Zr}$ and Ce-based MGs, the strain energy reserved in the strain-concentration striation is preferentially transported into the hyperelasticity region with the size of $r_{H}$. The strain-energy density in this hyperelasticity region is resultantly increased dramatically, which causes an adiabatic heating and a dilatation. A viscous flow region can be formed in the front of the crack tip, i.e., so-called fracture process zone. Driven by the shear stress, the crack tip advances into this "large" hyperelasticity region firstly, and then separates the fracture surface. As a sequence, the crack propagation leaves some viscous vein patterns on the fracture surface. For the Co- and $\mathrm{Mg}$-based MGs, the hyperelasticity region can be ignored. The strain energy stored in the strain-concentration striation is directly delivered to the crack tip. Without the influences of the hyperelasticity region, the crack tip penetrates into the glassy phase. Atomic bonds breaking is the main reason for consuming the strain energy transported from the surrounding linearly elastic region. Furthermore, as shown in Fig. 9, the strain-energy density is much higher than the fracture energy in the case of the Co- and Mg-based MGs. Without "buffering" from the hyperelasticity region, i.e., without the adiabatic heating and the dilatation consuming the strain energy, the only way to dissipate the large strain energy is speeding the crack propagation to be an unstably propagating level. The instability of the crack propagation initiates a bifurcation in the crack propagation, which then causes the multiple cracks [49]. This unstable crack propagation can bring out more fracture surfaces as compared to the case in stable crack propagation, such as the crack propagation in the $\mathrm{Zr}$ and Ce-based MGs. 
More fracture surfaces mean more fracture energy to be transported from the strain energy. Thus, the high strain energy stored in the Co- and Mg-based MGs can be dissipated by the multiple fracture surfaces, which are manifested as that the fractured Co- and Mg-based MGs are separated into several pieces.

It needs to be noted that the crack-bifurcation model is another theory elucidating the crack-propagation process [23]. When the crack propagation is speeded up to a critical velocity, the instable propagation of crack can generate a crack bifurcation [42]. In the present study, the observation and the calculation of the radius of the hyperelastic region, and the average width of strain-concentration striation are in the stage before the crack propagation. Therefore, the crack-bifurcation model is not used to elucidate the strain-energy transportation during the crack formation.

\section{Conclusion}

In this study, the correlation between the strain energy accumulation during the elastic deformation and the yielding behavior of MGs are investigated based on the DIC observation. The fracture behavior, i.e., the crack propagation is dominated by the balance between the strain energy stored in samples and the fracture energy. For the ductile MGs, before the crack propagation, a hyperelasticity region is formed in the front of crack. The strain energy transportation is buffered by the hyperelasticity region. The dynamic instability of crack propagation is frustrated, which brings out the shear fracture. For the brittle MGs, without buffering of the hyperelasticity region, the strain energy is transported directly to the fracture surface. Due to the absence of the energy consumption in the hyperelasticity region, the large amount of strain energy have to be consumed by the multiple fracture surfaces, which leads to the dynamic crack propagation. Our findings exhibit an unexplored strain-energy transportation process during the fracture process of MGs, which is possibly valid for other glassy systems that share similar structures, including the nano-glassy materials, and the oxidized glasses at the mesoscopic scale.

\section{Acknowledgement}

The work described in this paper was supported by grants from the MOST (No. 2015CB856800), NSF of China (Nrs. 51171098 and 51222102). 


\section{References}

[1] W. L. Johnson, MRS Bull. 24 (1999) 42.

[2] A. Inoue, Acta Mater. 48 (2000) 279.

[3] M.F. Ashby, A.L. Greer, Scr. Mater. 54 (2006) 321.

[4] J. Schroers, W.L. Johnson, Phys. Rev. Lett. 93 (2004) 255506.

[5] J. Das, M.B. Tang, K.B. Kim, R. Theissmann, F. Baier, W.H. Wang, J. Eckert, Phys. Rev. Lett. 94 (2005) 205501.

[6] X.K. Xi, D.Q. Zhao, M.X. Pan, W.H. Wang, Y. Wu, J.J. Lewandowski, Appl. Phys. Lett. 89 (2006) 181911.

[7] L.L. Zhang, R. Li, T. Xu, H.Y. Zhang, T. Zhang, Intermetallics, 52 (2014) 92.

[8] C.A. Schuh, A.C. Lund, Nature Mater. 2 (2003) 449.

[9] M.D. Demetriou, J.S. Harmon, M. Tao, G. Duan, K. Samwer, W.L. Johnson, Phys. Rev. Lett. 97 (2006) 065502.

[10]F. Spaepen, Acta Metall. 25 (1977) 407.

[11]Z. Wang, P. Wen, L.S. Huo, H.Y. Bai, W.H. Wang, Appl. Phys. Lett. 101 (2012) 121906.

[12]S.T. Liu, W. Jiao, B.A. Sun, W.H. Wang, J. Non-Cryst. Solids 376 (2013) 76.

[13]H.B. Ke, J.F. Zeng, C.T. Liu, Y. Yang, J. Mater. Sci. Technol. 30 (2014) 560.

[14]J. Ding, S. Patinet, M.L. Falk, Y.Q. Cheng, E. Ma, PNAS, 111 (2014) 14052.

[15]Z.Q. Liu, R.T. Qu, Z.F. Zhang, J. Appl. Phys. 117 (2015) 014901.

[16]R.T. Qu, H.S. Liu, Z.F. Zhang, Mater. Sci. Eng. A 582 (2013) 155.

[17]Z.Q. Liu, W.H. Wang, M.Q. Jiang, Z.F. Zhang, Philo. Mag. Lett. 94 (2014) 658.

[18]J.J. Lewandowski, W.H. Wang, A.L. Greer, Philo. Mag. Lett. 85, (2005) 77.

[19]M.Q. Jiang, Z. Ling, J.X. Meng, L.H. Dai, Philo. Mag. 88, (2008) 407.

[20]Z.Q. Liu, R.T. Qu, Z.F. Zhang, J. App. Phys.. 117, (2015) 014901.

[21]B.A. Sun, W.H. Wang, Prog. Mater Sci. 74, (2015) 211.

[22]Z.F. Zhang, G. He, J. Eckert, L. Schultz, Phys. Rev. Lett. 91, (2003) 045505.

[23]J. Fineberg. The dynamics of rapidly moving tensile cracks in brittle amorphous material.

Dynamic fracture mechanics, World Scientific, Hong Kong, 2006.

[24]A. Livne, E. Bouchbinder, I. Svetlizky, J. Fineberg. Science, 327 (2010) 1359.

[25]E. Bouchbinder, A. Livne, J. Fineberg, Phys. Rev. Lett. 101 (2008) 264302.

[26]T. Baumberger, O. Ronsin, Eur. Phys. J. E, 31 (2010) 51. 
[27]X.J. Dai, F.J. Yang, Z.N. Chen, X.X. Shao, X.Y. He, Opt. Laser Eng. 65 (2015) 64.

[28]M.A. Sutton, J.J. Orteu, H.W. Schreier. Image correlation for shape, motion and deformation measurements, Springer, New York, 2009.

[29]S.H. Joo, H. Kato, K. Gangwar, S. Lee, H.S. Kim, Itemetalics. 32 (2013) 21.

[30]J. Zhang, P. Aimedieu, F. Hild, S. Roux, T. Zhang, Scripta Mater. 61 (2009) 1145.

[31]Y. Wu, H. Bei, Y.L. Wang, Z.P. Lu, E. P. George, Y.F. Gao, International Journal of Plasticity $71(2015) 136$.

[32]Y. Sun, E.N. Hoffman, P.S. Lam, X.D. Li, Scipta Mater. 65 (2011) 388.

[33]Y. Belrhiti, O. Pop, A. Germaneau, P. Doumalin, J.C. Dupre, H. Harmuth, M. Huger, T. Chotard, J. Eur. Ceram. Soc. 35 (2014) 823.

[34]H.P. Song, H. Zhang, Y.L. Kang, G.Y. Huang, D.H. Fu, C.Y. Qu, Tectonophysics. 608 (2013) 1343.

[35]M. Eskandari, M.R. Yadegari-Dehnavi, A. Zarei-Hanzaki, M.A. Mohtadi-Bonab, R. Basu, J.A. Szpunar, Opt. Laser Eng. 67 (2015) 1.

[36]Y. Belrhiti, O. Pop, A. Germaneau, P. Doumalin, J.C. Dupre, H. Harmuth, M. Huger, T. Chotard, J. Eur. Ceram. Soc. 35 (2014) 823.

[37]H.P. Song, H. Zhang, Y.L. Kang, G.Y. Huang, D.H. Fu, C.Y. Qu. Tectonophysics 608 (2013) 1343.

[38]B. Zhang, D.Q. Zhao, M.X. Pan, R.J. Wang, W.H. Wang. Acta Mater. 54 (2006) 3025.

[39]Y. Kawamura, T. Shibata, A. Inoue, T. Masumoto. Appl. Phys. Lett. 69 (1996) 1208.

[40]R.J. Atkin, N. Fox, An Introduction to the Theory of Elasticity, Longman, London, 1980.

[41]Z. Han, W.F. Wu, Y. Li, Y. J. Wei, H.J. Gao. Acta Mater. 57 (2009) 1367.

[42]B. Lawn. Fracture of brittle solids, 2nd ed, Cambridge University Press, Cambridge, 1993.

[43]G. Wang, K.C. Chan, X.H. Xu, W.H. Wang, Acta Mater. 56 (2008) 5845.

[44]G. I. Taylor, Proc. R. Soc. A 201 (1950) 192.

[45]J.R. Rice, N. Levy, Physics of Strength and Plasticity, MIT Press, Cambridge, MA, 1969.

[46]M.J. Buehler, H.J. Gao, Dynamic fracture mechanics, World Scientific, Hong Kong, 2006.

[47]B. Lawn, Fracture of Brittle Solids, 2nd ed., Cambridge University Press, Cambridge, 1993.

[48]M.J. Buehler, F.F. Abraham, H.J. Gao. Nature 426 (2003) 141.

[49]M.J. Buehler, H.J. Gao. Nature 439 (2006) 307.

[50]J. Fineberg. Dynamic fracture mechanics, World Scientific, Hong Kong, 2006. 
[51]B. Yang, M.L. Morrison, P.K. Liaw, R.A. Buchanan, G.Y. Wang, C.T. Liu, M. Denda, Appl. Phys. Lett. 86 (2005) 141904. 


\section{Figure captions}

Fig. 1 XRD patterns of the four MGs.

Fig. 2 Nominal compression stress-strain curves of the four MGs.

Fig. 3 Fractographies of the four MGs. (a) Lateral surface of the fractured Zr-based MG. (b) Fracture surface of the fractured Zr-based MG. The inset shows some shear bands that are pointed at by arrows. (c) Lateral surface of the fractured Ce-based MG. (d) Fracture surface of the fractured Ce-based MG. The inset shows some shear bands that are pointed at by arrows. (e) Lateral surface of the fractured Mg-based MG. (f) Fracture surface of the fractured Mg-based MG. (g) Lateral surface of the fractured Co-based MG. (h) Fracture surface of the fractured Co-based MG.

Fig. 4 DIC images of strained Zr-based MG. (a) Speckle image of the Zr-based MG without loading. (b) Contour maps of the $\varepsilon_{x \max }$ field. (c) Contour maps of the $\varepsilon_{y \max }$ field. $\gamma$ points to the width of strain-concentration striation. (d) Contour maps of the $\gamma_{\max }$ field.

Fig. 5 DIC images of the strained Ce-based MG. (a) Speckle image of the Ce-based MG without loading. (b) Contour maps of the $\varepsilon_{x \max }$ field. (c) Contour maps of the $\varepsilon_{y \max }$ field. $\gamma$ points to the width of strain-concentration striation. (d) Contour maps of the $\gamma_{\max }$ field.

Fig. 6 DIC images of the strained Co-based MG. (a) Speckle image of the Co-based MG without loading. (b) Contour maps of the $\varepsilon_{x \max }$ field. (c) Contour maps of the $\varepsilon_{y \max }$ field. $\gamma$ points to the width of strain-concentration striation. (d) Contour maps of the $\gamma_{\max }$ field.

Fig. 7 DIC images of the strained Mg-based MG. (a) Speckle image of the Mg-based MG without loading. (b) Contour maps of the $\varepsilon_{x \max }$ field. (c) Contour maps of the $\varepsilon_{y \max }$ field. $\gamma$ points to the width of strain-concentration striation. (d) Contour maps of the $\gamma_{\max }$ field.

Fig. 8 Profile of the distributions of the maximum $\varepsilon_{x \max }, \varepsilon_{y \max }$ and $\gamma_{\max }$ values at different stresses of the four MGs on the maximum shear stress plane, i.e., along the dash lines in Fig. 4-7. (a), (b) and 
(c) correspond to the distribution of the maximum $\varepsilon_{x \max }, \varepsilon_{y \max }$ and $\gamma_{\max }$ values of the $\mathrm{Zr}$-based MG, respectively. (d), (e) and (f) correspond to the distribution of the maximum $\varepsilon_{x \max }, \varepsilon_{y \max }$ and $\gamma_{\max }$ values of the Ce-based MG, respectively. (g), (h) and (i) correspond the distribution of the maximum $\varepsilon_{x \max }, \varepsilon_{y \max }$ and $\gamma_{\max }$ values of the Co-based MG, respectively. (j), (k) and (l) correspond the distribution of the maximum $\varepsilon_{x \max }, \varepsilon_{y \max }$ and $\gamma_{\max }$ values of the $\mathrm{Mg}$-based MG, respectively. The dark-solid lines cover the integrating area covered by the strain-concentration striation peak.

Fig. 9 Sketch for the concepts of the hyperelasticity region and the characteristic length for energy flux. (a) Sketch for the case that the hyperelasticity region is dominative, i.e., the softening behavior. (b) Sketch for the case that the linear elastic region is dominative, i.e., the stiffening behavior.

Fig. 10 Comparative plots between the fracture energy $(I)$ and the strain energy density $(S)$ of the four MGs. Open circles point to the fracture energies at different crack propagation speeds of the four MGs. Dash lines point to the strain energy density calculated by Eq. (4). 
Table I Mechanical properties of the four MGs, i.e., stress intensity factor $(K)$, Poisson's ratio $(v)$, bulk modulus $(B)$, shear modulus $(G)$, Young's modulus $(E)$, yield strength $\left(\sigma_{Y}\right)$, plastic strain $\left(\varepsilon_{p}\right)$, and shear wave speed $\left(c_{s}\right)$.

\begin{tabular}{ccccccccc}
\hline Sample & $\begin{array}{c}K \\
\left(\mathrm{MPa} \cdot \mathrm{m}^{0.5}\right)\end{array}$ & $v$ & $\begin{array}{c}B \\
(\mathrm{GPa})\end{array}$ & $\begin{array}{c}G \\
(\mathrm{GPa})\end{array}$ & $\begin{array}{c}E \\
(\mathrm{GPa})\end{array}$ & $\begin{array}{c}\sigma_{Y} \\
(\mathrm{MPa})\end{array}$ & $\varepsilon_{p}$ & $\begin{array}{c}c_{s} \\
(\mathrm{~m} / \mathrm{s})\end{array}$ \\
\hline Zr-based MG & $42.6 \pm 9.9$ & $0.370 \pm 0.009$ & $114.1 \pm 6.9$ & $32.3 \pm 0.9$ & $88.6 \pm 6.4$ & $1749 \pm 36$ & $0.014 \pm 0.003$ & $2191 \pm 39$ \\
\hline Ce-based MG & $5.7 \pm 2.8$ & $0.340 \pm 0.012$ & $31.8 \pm 3.8$ & $11.4 \pm 1.2$ & $30.5 \pm 4.9$ & $403 \pm 21$ & $0.014 \pm 0.002$ & $1277 \pm 13$ \\
\hline Co-based MG & $5.0 \pm 2.7$ & $0.338 \pm 0.011$ & $248.2 \pm 11.9$ & $90.0 \pm 1.5$ & $240.1 \pm 14.3$ & $4763 \pm 42$ & $0.006 \pm 0.002$ & $3128 \pm 9$ \\
\hline Mg-based MG & $1.3 \pm 0.6$ & $0.310 \pm 0.013$ & $45.1 \pm 4.6$ & $19.3 \pm 1.0$ & $50.6 \pm 3.2$ & $895 \pm 29$ & 0 \\
\hline
\end{tabular}




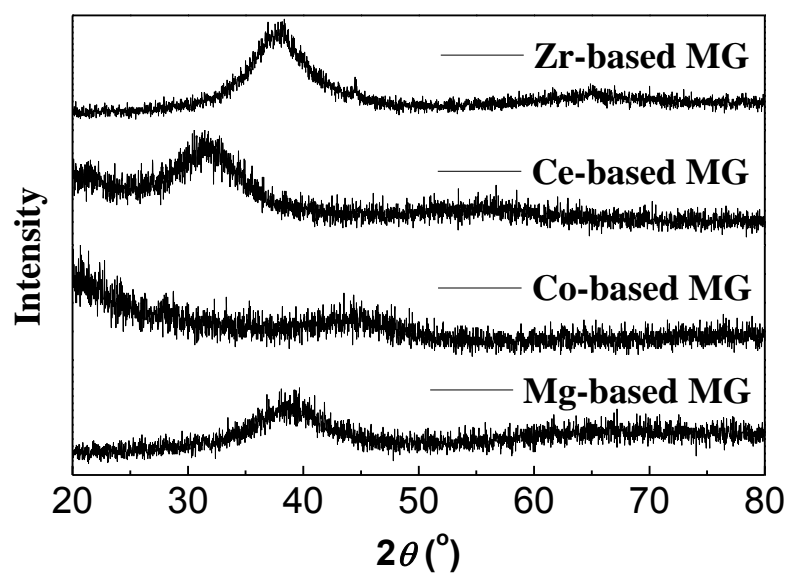

Fig. $1 \mathrm{~J}$. Li, et al. 

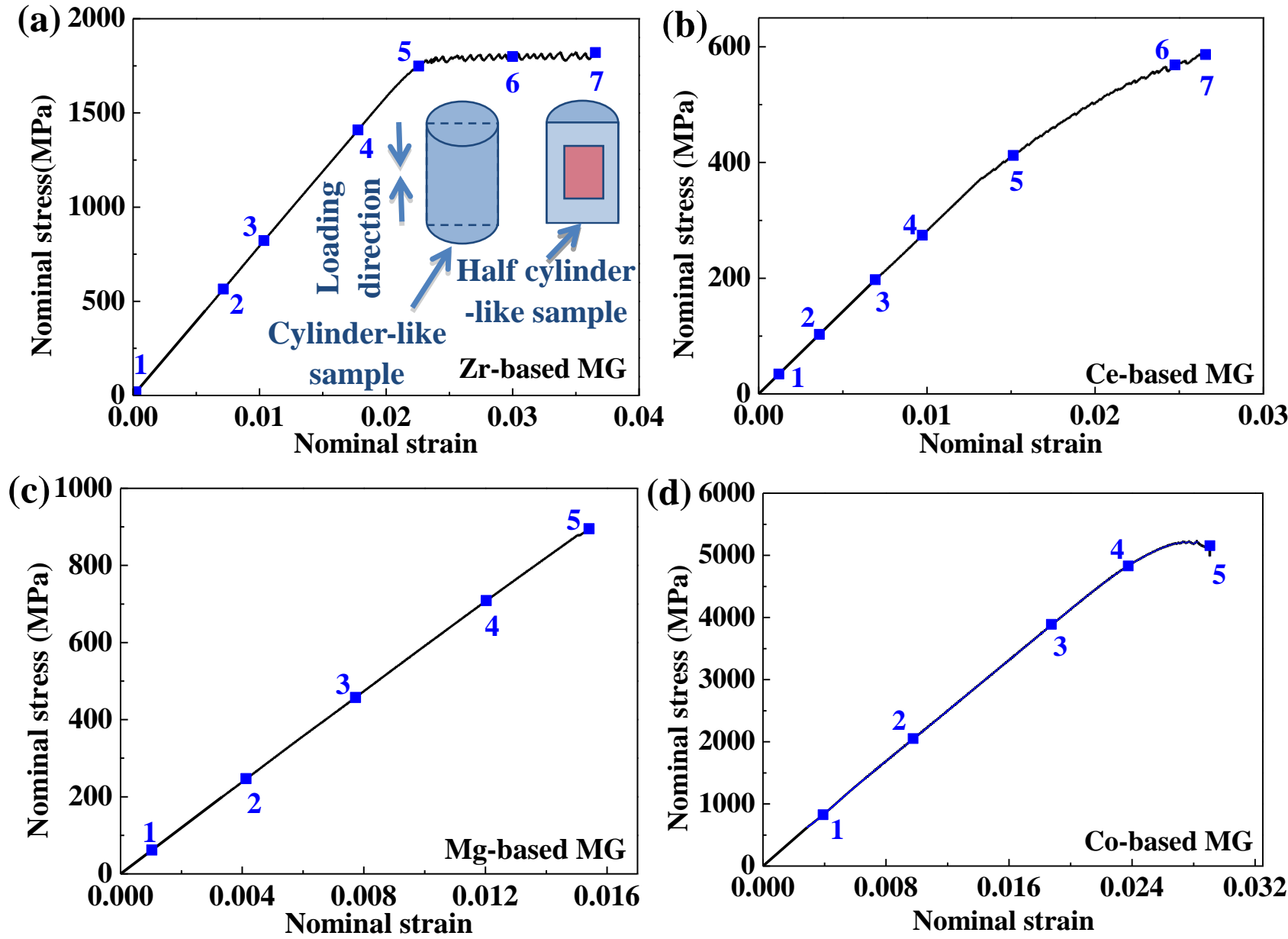

Fig. 2 J. Li, et al. 


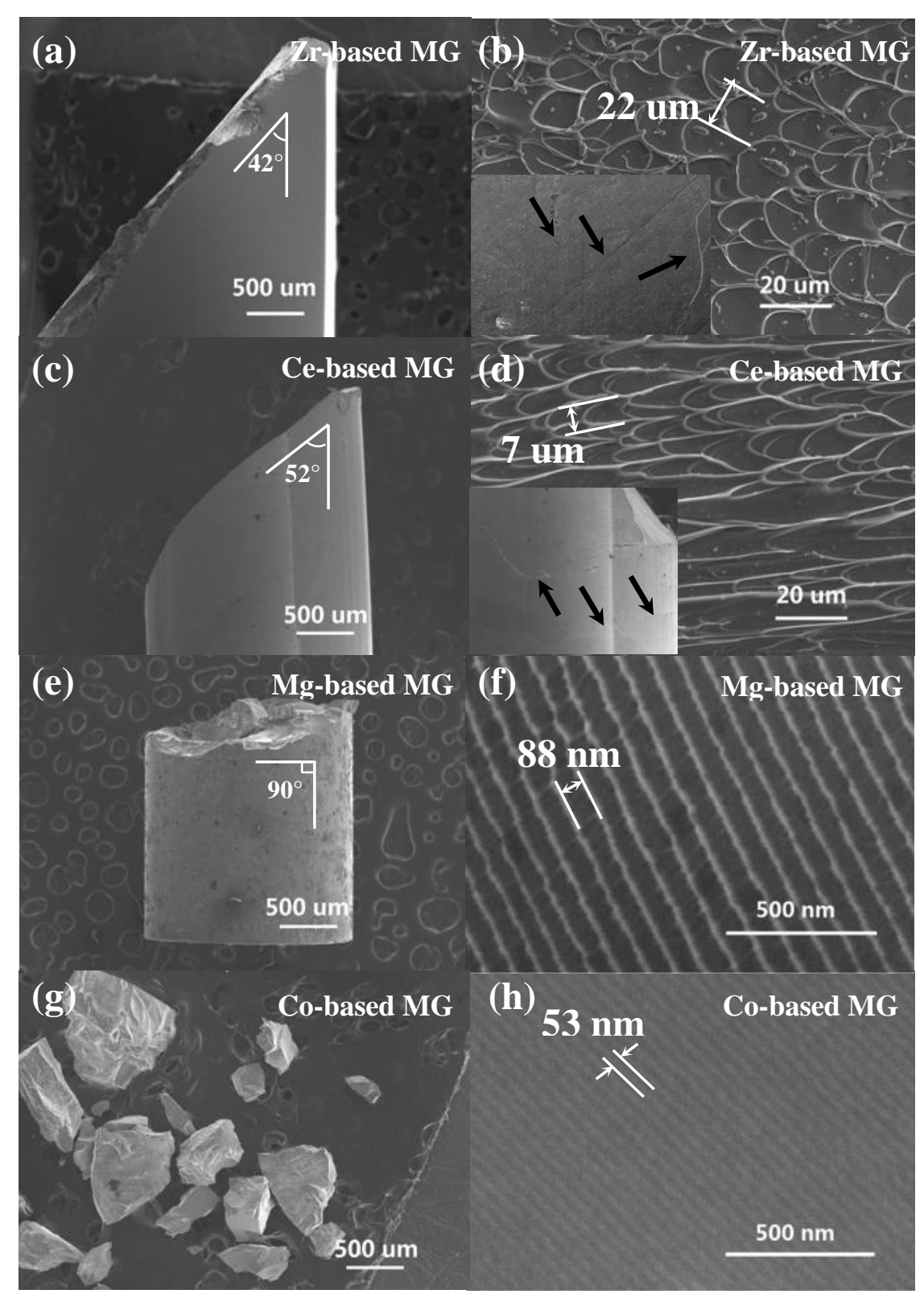

Fig. 3 J. Li, et al. 

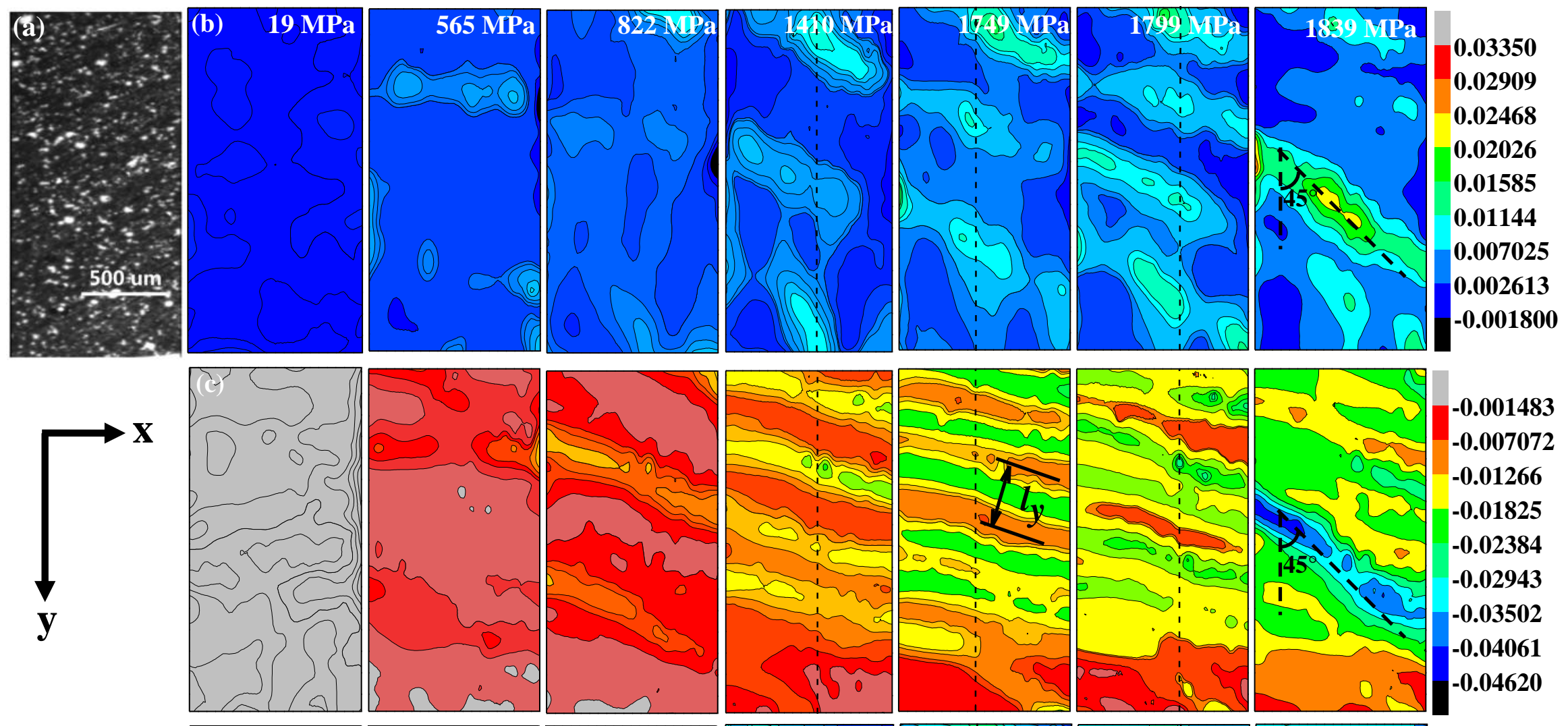

$-0.001483$

$-0.007072$

$-0.01266$ $-\mathbf{0 . 0 1 8 2 5}$

$-\mathbf{0 . 0 2 3 8 4}$

$-0.02943$

$-0.03502$

$-0.04061$

$-0.04620$
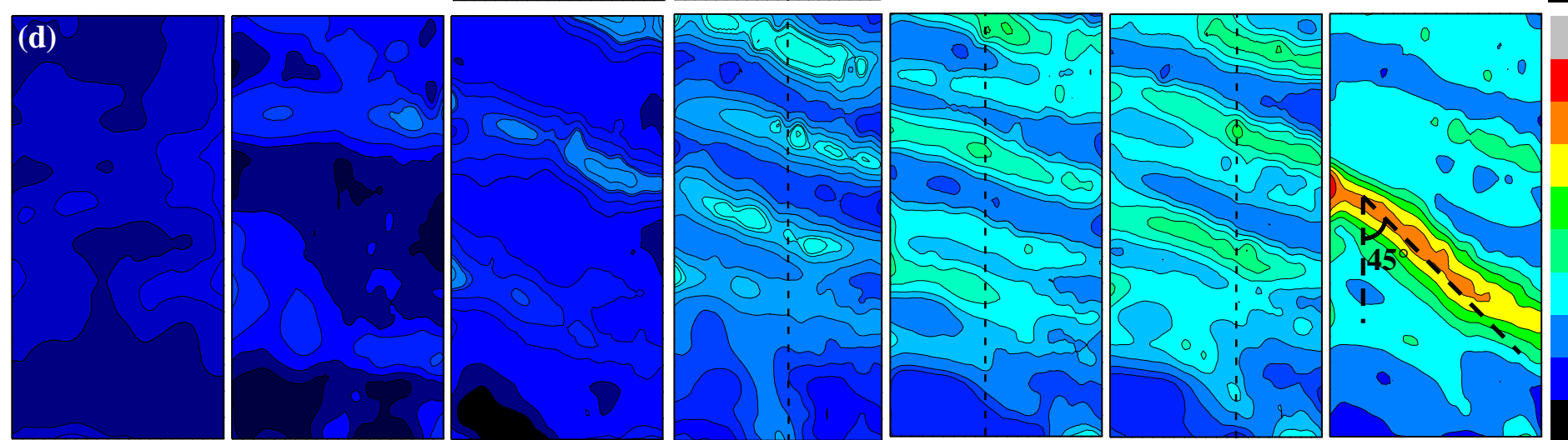

0.06920

0.06105

0.05290

0.04475

0.03660

0.02845

0.02030

0.01215

0.004000

Fig. $4 \mathrm{~J}$. Li, et al. 


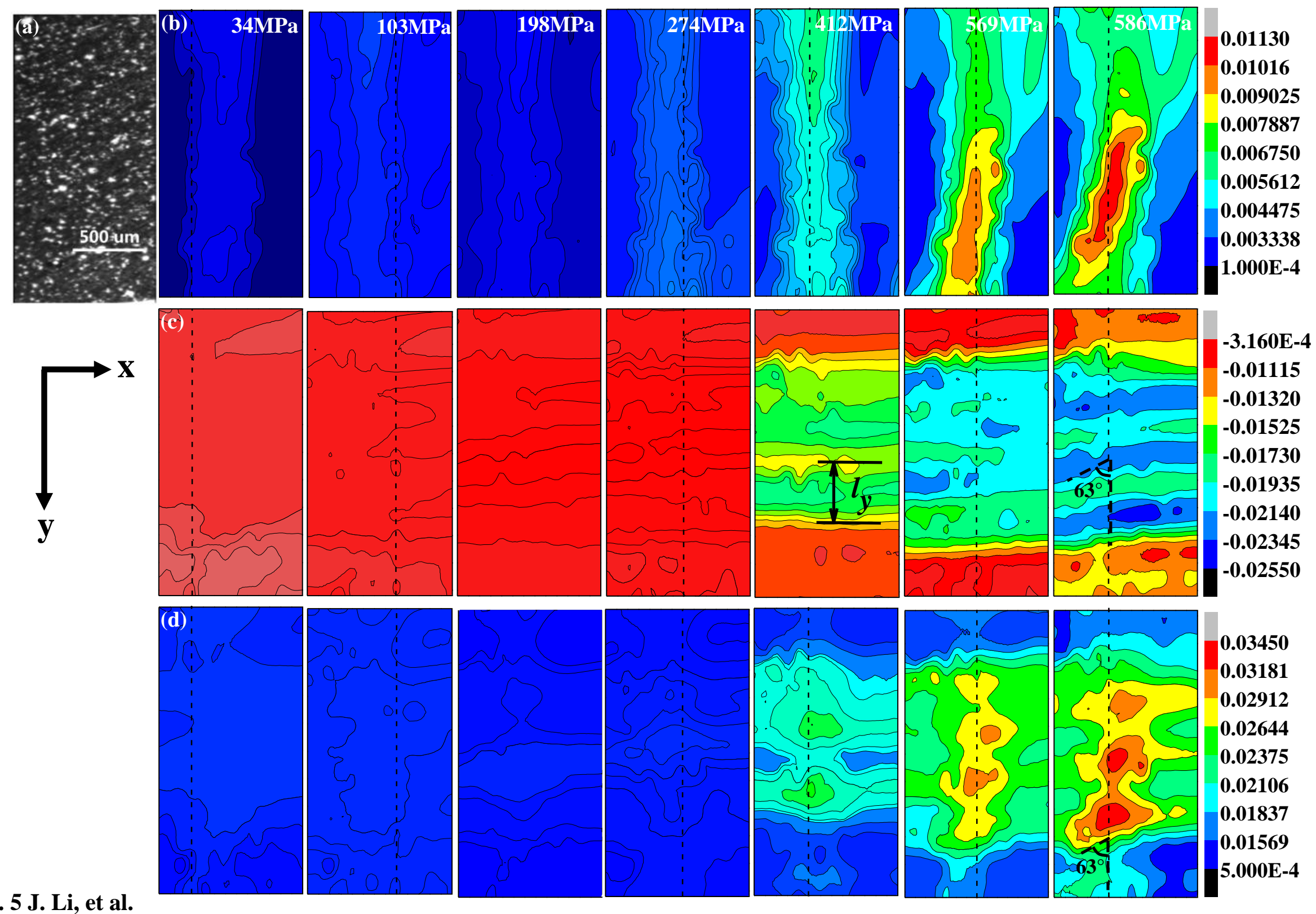

Fig. 5 J. Li, et al. 

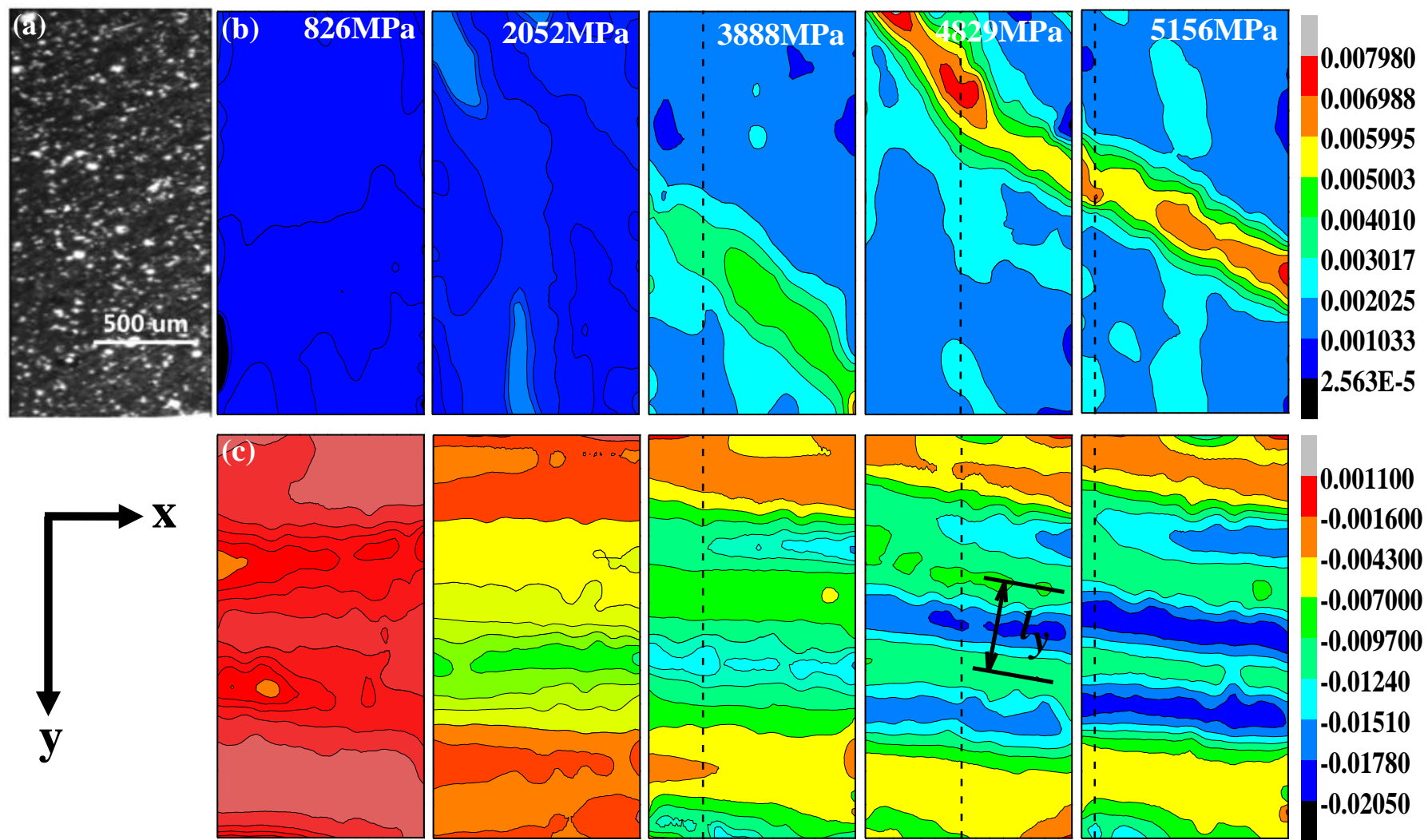

0.001100

$-0.001600$

$-0.004300$

$-\mathbf{0 . 0 0 7 0 0 0}$

$-0.009700$

$-0.01240$

$-0.01510$

$-0.01780$

$-0.02050$
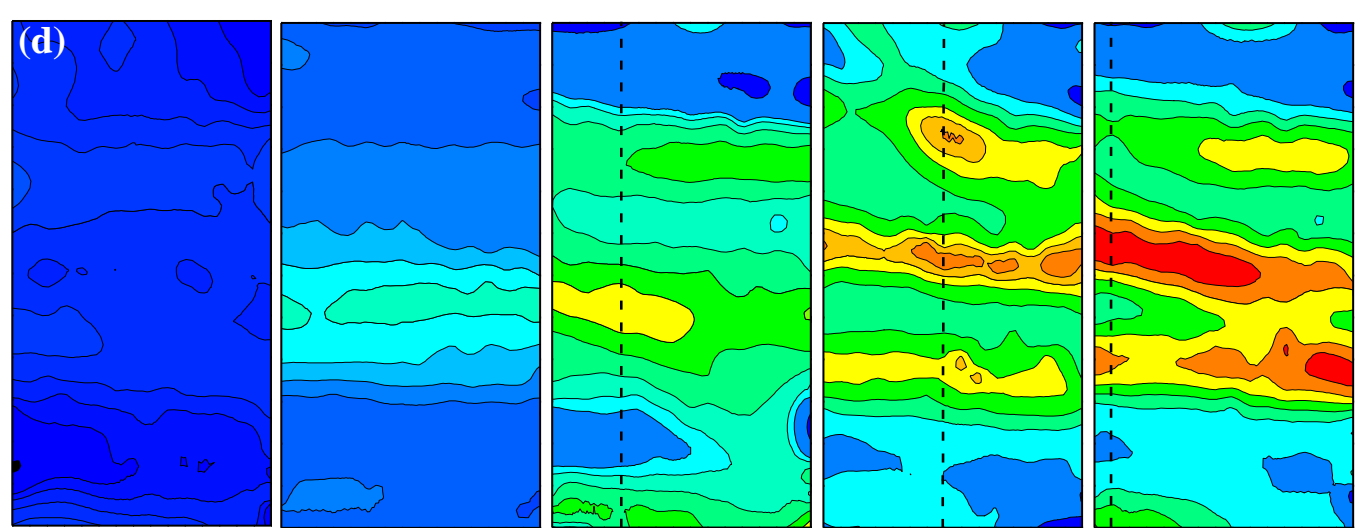

0.02660

0.02338

0.02015

0.01693

0.01370

0.01048

0.007250

0.004025

$3.850 \mathrm{E}-4$

Fig. 6 J. Li, et al. 


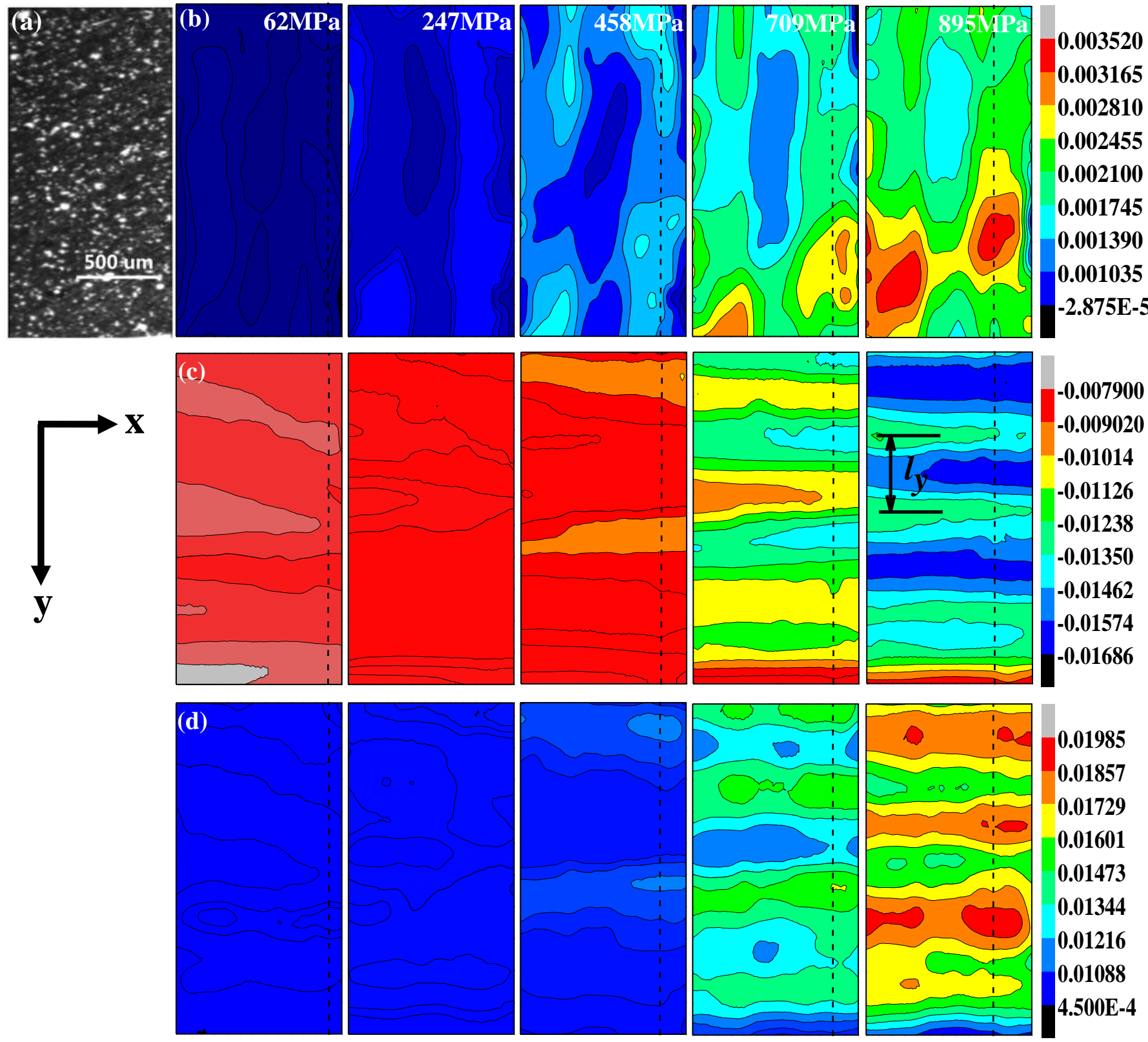

Fig. 7 J. Li, et al. 
(a)

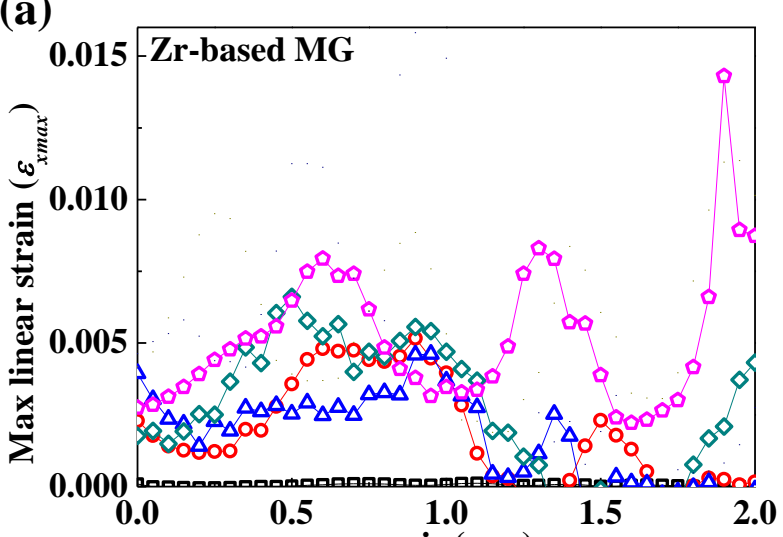

(d)

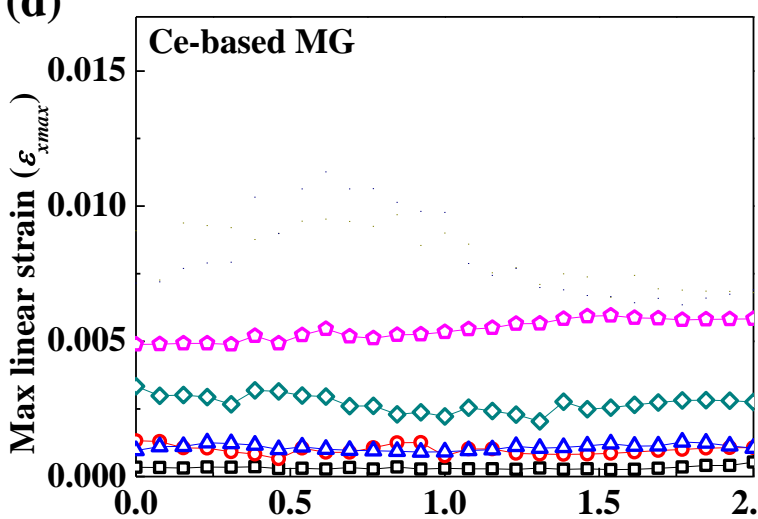

(b) 0.000

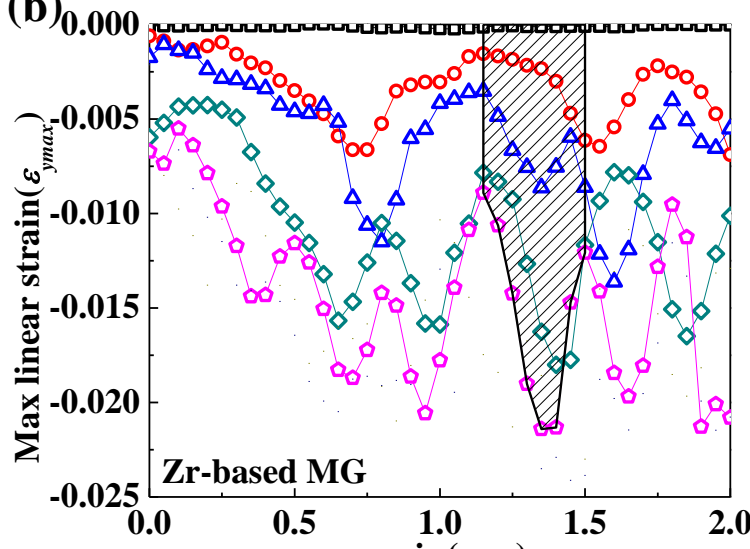

(e) 0.000

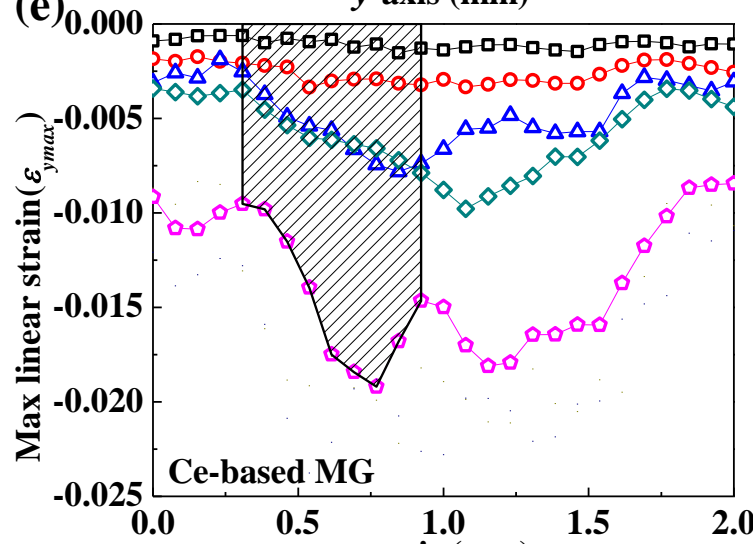

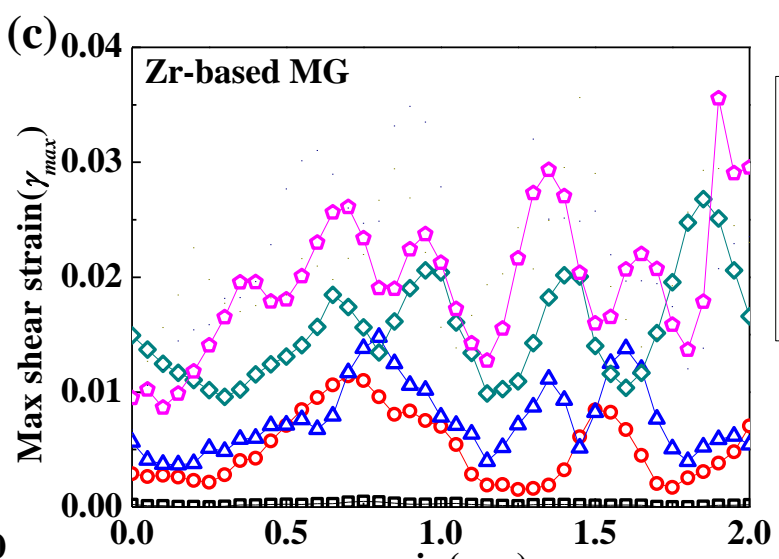

\section{- 19MPa \\ $-0-565 \mathrm{MPa}$ \\ $-\triangle 822 \mathrm{MPa}$ \\ $\checkmark 1410 \mathrm{MPa}$ \\ - 1749MPa}

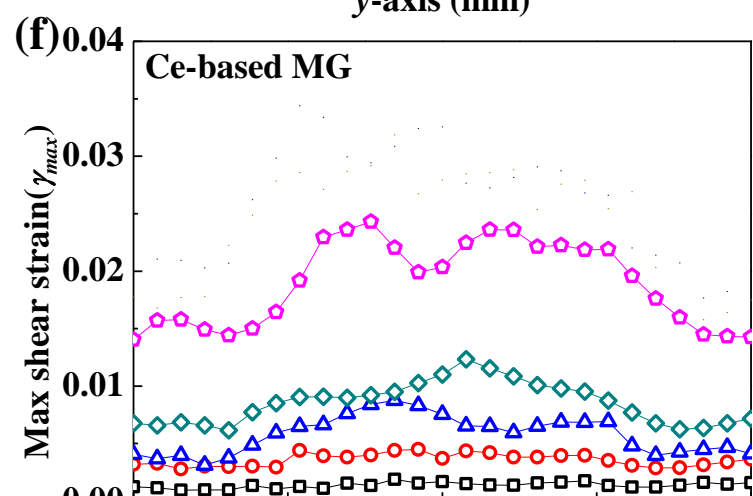
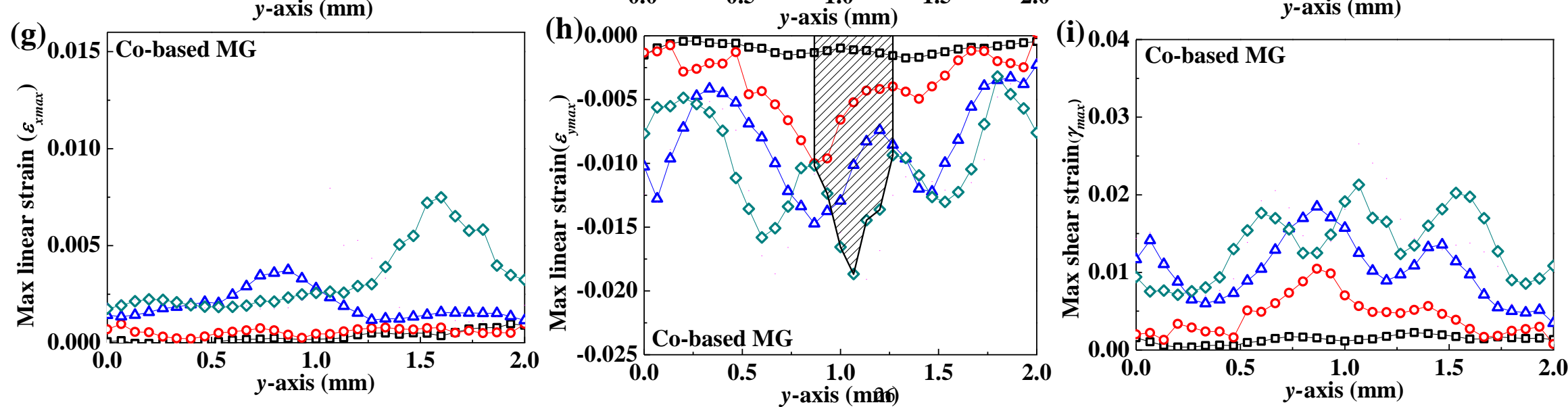

- - 34MPa

- $-103 \mathrm{MPa}$

$\triangle-198 \mathrm{MPa}$

$\checkmark 274 \mathrm{MPa}$

$-\square-403 M P a$

-o- 2052MPa

$-\triangle$ 3888MPa

$\checkmark 4829 \mathrm{MPa}$

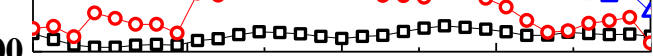
$y$-axis (mm) 

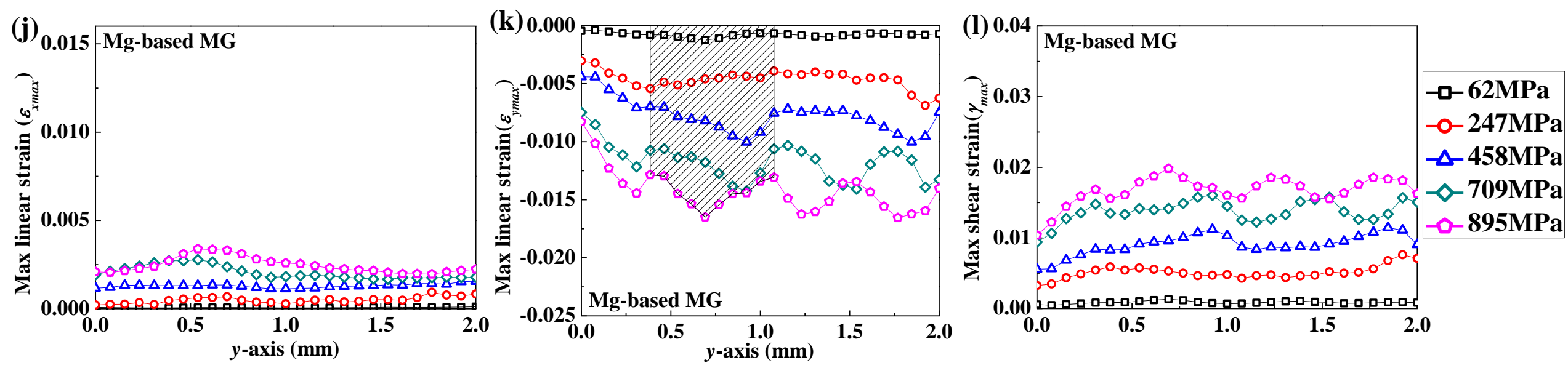

Fig. $8 \mathrm{~J}$. Li, et al. 

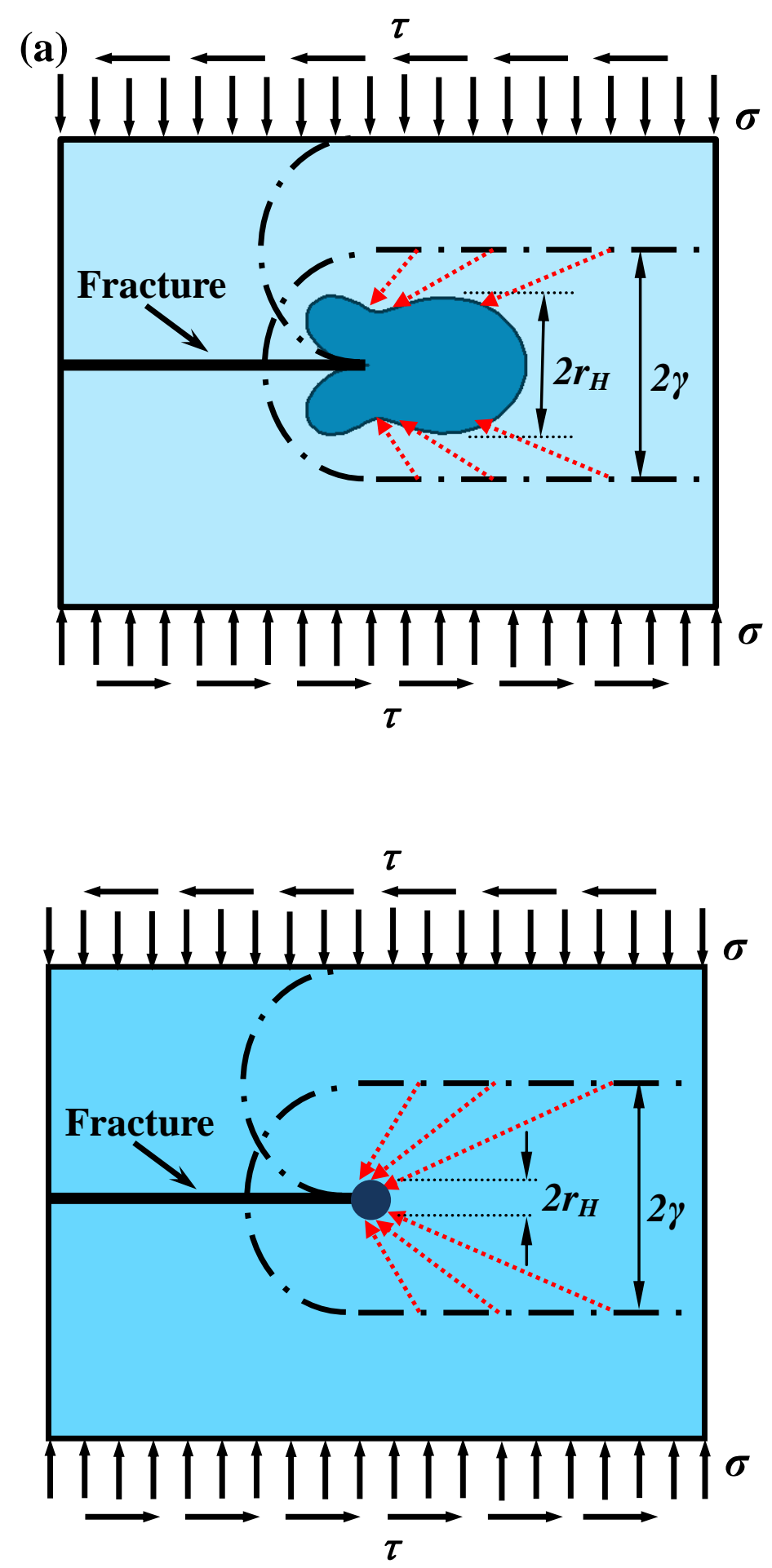

Fig. 9 J. Li, et al. 


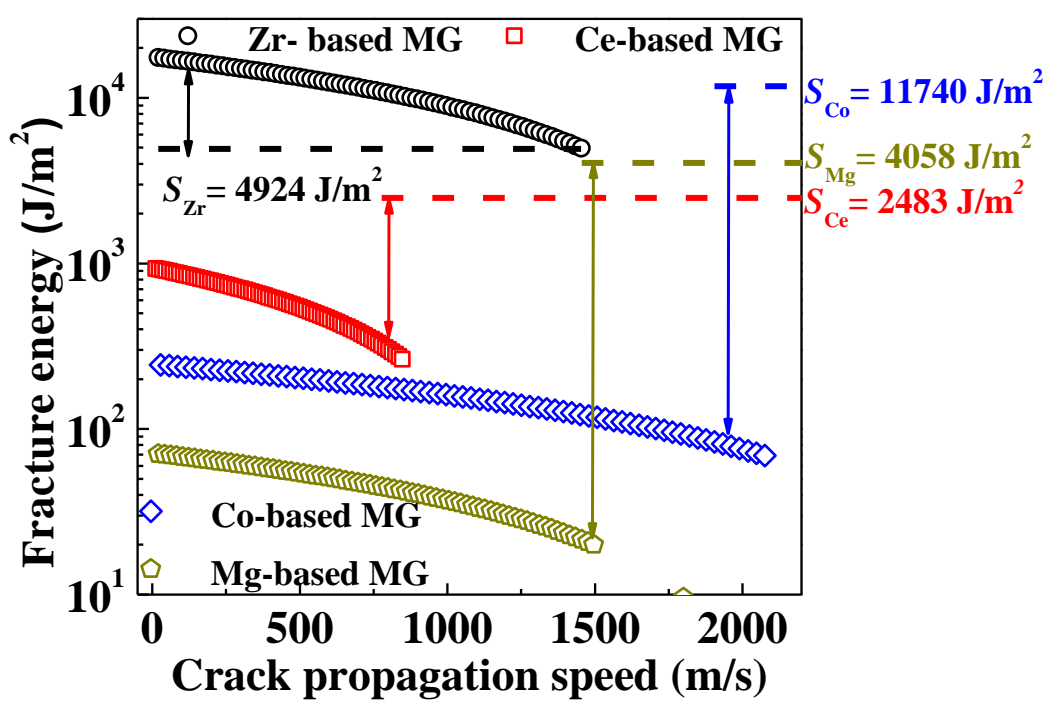

Fig. 10 J. Li, et al. 